FERMILAB-TM-1248-ESH-REV

\title{
MEASUREMENT OF RADIATION QUALITY FACTORS USING A RECOMBINATION CHAMBER
}

\author{
Revised June 2009
}

\author{
J. Donald Cossairt and Michael A. Gerardi
}

\section{Need for This Revision}

In 2007 the Department of Energy modified the system of radiation dosimetry to be used for occupational radiation protection at its facilities in its regulation on occupational radiation protection, 10 CFR Part 835. The previous system in place prior to 2007 had emerged in approximately 1973 and so can be called the "1973 Radiation Protection System". The system instituted by DOE in 2007 was originally developed by the International Commission on Radiological Protection (ICRP) in 1990 and so might be called the "1990 Radiation Protection System". The principal impact on this change at particle accelerators is in the quantities used to measure radiation dose to personnel exposed to particulate radiation, most prominently neutrons. A detailed discussion of this topic has been provided by Cossairt and Vaziri (Co09).

The original version of TM-1248 by J. D. Cossairt, D. W. Grobe, and M. A. Gerardi, appended here, discussed the details of the recombination chamber technique as employed in the determination of radiation quality factors $(Q F s)$ in radiation fields found at Fermilab. Since that time, the technique has been used routinely and selected results published by Cossairt et al. [(Co85a), (Co85b), (Co87)] and Elwyn and Cossairt (E186). The results presented in the original version of TM-1248 were, of course, reflective of the 1973 Radiation Protection System. Briefly, the normalized response $I$ of the special ion chamber used for these measurements as a function of applied bias voltage $V$ in a given radiation field has the following dependence:

$$
I=k V^{N}
$$

where $k$ is a constant. It turns out that the value of $N$ is physically dependent on the ionization density in the chamber. This phenomenon thus ties it to the radiation quality factor $Q F$, a quantity also tied to ionization density since it is defined as a function of linear energy transfer (LET) in tissue. In the work described by the original TM, six mixed radiation fields were staged

using ${ }^{60} \mathrm{Co}$ and ${ }^{238} \mathrm{PuBe}$ sources each with calculated values of $Q F$. The chamber response for each such field was measured and a value of $N$ determined (see Fig. 1 of the original TM). Figure 2 of the original TM shows the results of correlating $N$ with $Q F$. The relationship is describable by both linear and power law fits to the mixed field measurements and those are also provided.

It is timely to review these results in light of the change to the 1990 Radiation Dosimetry System. As reported by Cossairt and Vaziri (Co09), the quantity $Q F$ is replaced with that of the radiation weighting factor $w_{R}$. However, this change in terminology is not fully implemented and the scientific literature to date is inconsistent. Thus the term $Q F$ remains prominently in use. To limit confusion in this revised TM, $w_{R}$ will be used here to refer to the 1990 Radiation Protection System quantity. 


\section{New Results}

Unfortunately, the mixed field measurements used to establish the functional dependence of $N$ on $w_{R}$ cannot be repeated since the ${ }^{238} \mathrm{Pu}-\mathrm{Be}$ source set used before is now no longer present at Fermilab. Also, the original measurements were conducted in one of the upstairs bedrooms of the Site 68 farmhouse, a structure that had solid, not hollow, brick walls. Unfortunately, this structure no longer exists. Thus, it was deemed expedient to reanalyze the existing data.

Based on the work of Höfert and Raffnsøe (Ho80), in the original measurements reported in 1984 the value of $Q F$ of the radiation field of the ${ }^{238} \mathrm{PuBe}$ source was taken to be 6.9 , if one includes the photon component of the neutron-dominated radiation field. An understanding of the strength of the photon component is thus needed. A measurement by Lawrence (La62) found that in the radiation field of a ${ }^{239} \mathrm{PuBe}$ source, $92 \pm 5 \%$ of the absorbed dose was due to the neutrons with the remaining $8 \pm 1 \%$ due to the photons always present in such a radiation field. Absorbed dose is nearly ideal as the metric for the relative neutron and photon components since it is a physical quantity (the energy deposited per unit mass) that is, in principle, directly measurable.

Cossairt and Vaziri (Co09) found an average value of $w_{R}=12.4$ for the neutron component of a standard ${ }^{241}$ AmBe neutron under the 1990 Radiation Protection System. Applying Lawrence's results to this value and understanding that such a recombination chamber essentially measures absorbed dose directly when biased at its saturation voltage, one finds a value of $w_{R}=11.49$ for the overall radiation field including the photons. One can thus calculate values of $w_{R}$ under the 1990 Radiation Dosimetry System to correlate with the six measured values of $N$ reported in the original TM. Doing this and providing the same types of linear and power low fits as was done before, one obtains Fig. 1A showing new results under the 1990 Radiation Dosimetry System.

\section{Sources of Error}

In the above it is clear that three different types of $\alpha$-neutron sources are discussed; ${ }^{238} \mathrm{PuBe}$, ${ }^{239} \mathrm{PuBe}$, and ${ }^{241} \mathrm{AmBe}$. The reported differences between the radiation fields generated by these three sources are customarily regarded as minimal but perhaps a bit more explanation of this is needed. This assertion is reasonable because the $\alpha$-particle decay energies of these three $\alpha$ emitting radionuclides are quite similar, just above 5.0 MeV. Naturally-occurring beryllium is $100 \%{ }^{9} \mathrm{Be}$. The neutrons and photons are emitted by means of the ${ }^{9} \mathrm{Be}(\alpha, \mathrm{n}){ }^{12} \mathrm{C}$ nuclear reaction. The nuclear reaction $Q_{\text {value }}=5.702 \mathrm{MeV}$ so neutrons having kinetic energies of up to just above $11 \mathrm{MeV}$ for all of these sources are produced. As seen in the published literature, some of which is cited in (Co09), the nuclear excited states of ${ }^{12} \mathrm{C}$ are clearly reflected in the energy spectrum of the emitted neutrons, with peak-broadening due to ionization losses by the $\alpha$-particles in the source matrix. It is thus believed that considering these three types of $\alpha$-neutron sources to be identical is reasonable for present purposes.

The value of $Q F=6.9$ under the 1973 Radiation Dosimetry System used in the original version of the TM was taken from Höfert and Raffnsøe (Ho80). These authors cite no references as to the origin of this value. Papers by Kemmochi give slightly lower values of 6.56 (Ke78a) or 6.5 (Ke78b) for the $Q F$ of such $\alpha$-neutron sources. On the other hand, applying the results of Lawrence (La60) to the ${ }^{241} \mathrm{AmBe}$ spectrum evaluated under the 1973 Radiation Dosimetry 
System by Cossairt and Vaziri (Co09) to have $Q F=8.17$ for the neutron-only component, one gets $Q F=7.6$ for the overall radiation field. Thus an uncertainty of this order is present in the measurements used to generate the $N-Q F$ and $N-w_{R}$ dependencies that are proportional to the absorbed dose of the neutron component of the staged mixed field.

Since the original measurements were conducted in the upstairs of the brick farmhouse, the neutron spectrum plausibly could have been perturbed by backscattering from the walls. However comparison with other published results for recombination chambers shows good qualitative agreement [see (Pa73), (Su84)]. Also, several radiation fields at Fermilab have been studied where it was possible to compare results using the recombination chamber technique with those obtained through neutron spectrum measurements, an entirely independent method [(Co85a), (El86), (Co87)]. The results are consistent, within other errors present.

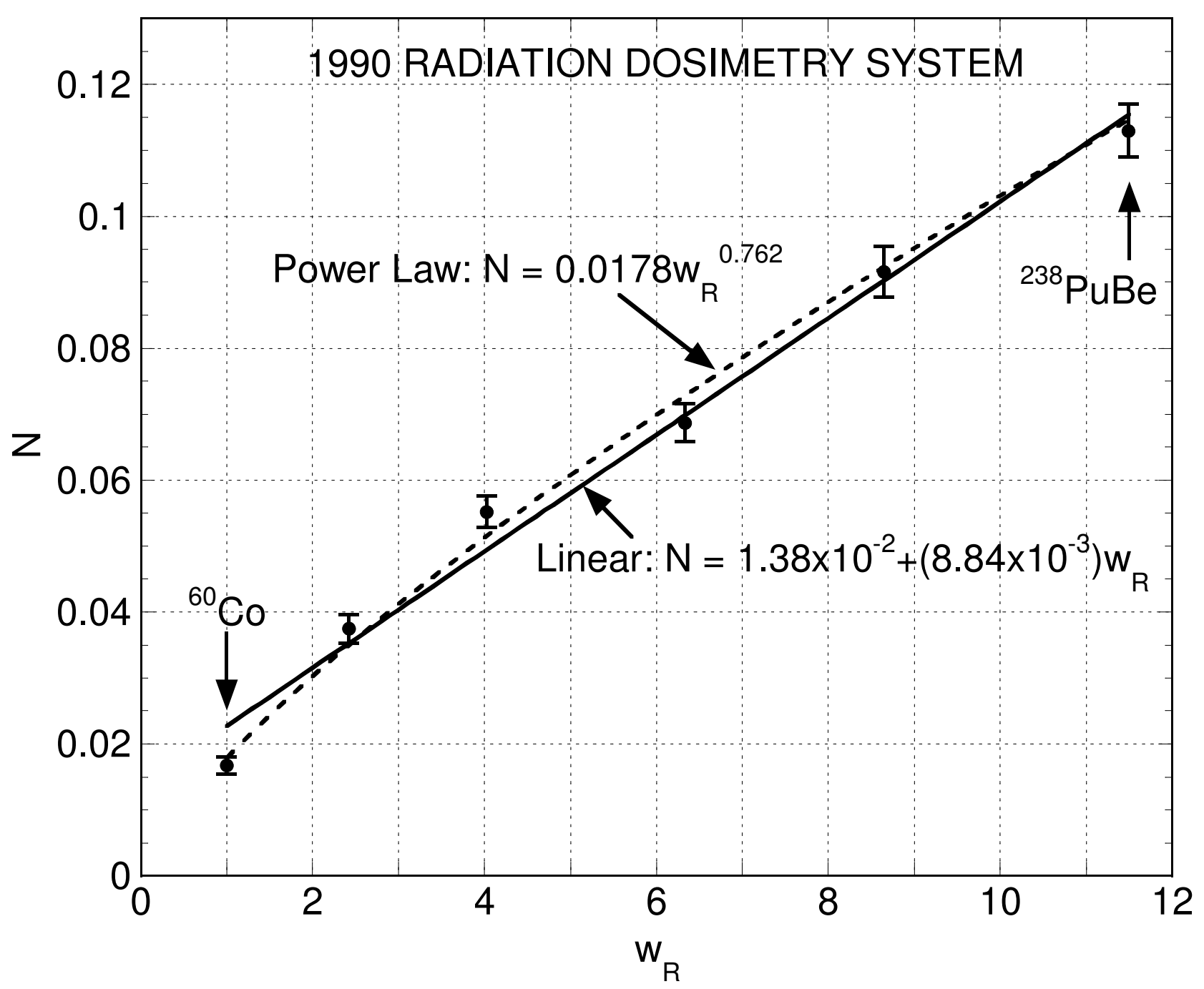

FIGURE 1A 


\section{ADDITIONAL REFERENCES FOR THE JUNE 2009 REVISION}

(Co85a) J. D. Cossairt, J. G. Couch, A. J. Elwyn, and W. S. Freeman, "Radiation measurements in a labyrinth penetration at a high-energy proton accelerator", Health Phys. $\underline{49}$ (1985) 907-917.

(Co85b) J. D. Cossairt and L. V. Coulson, "Neutron skyshine measurements at Fermilab", Health Phys. $\underline{48}$ (1985) 171-181.

(Co87) J. D. Cossairt and A. J. Elwyn, "Personal dosimetry in a mixed field of highenergy muons and neutrons", Health Phys. $\underline{52}$ (1987) 813-818.

(Co09) J. D. Cossairt and K. Vaziri, "Neutron dose per fluence and weighting factors for use at high energy accelerators", Health Phys. $\underline{6}$ (2009) 617-628.

(E186) A. J. Elwyn and J. D. Cossairt, "A study of neutron leakage through an Fe shield at an accelerator", Health Phys. 51 (1986) 723-735.

(Ke78a) M. Kemmochi, "A study on the REM ratemeter for direct reading of the dose equivalent rate", Health Phys. $\underline{34}$ (1978) 361-370.

(Ke78b) M. Kemmochi, "A rem ratemeter for mixed radiation using two spherical ionization chambers", Health Phys. $\underline{35}$ (1978) 333-340.

(La62) D. C. Lawrence, "Mixed radiation dosimetry of a plutonium-beryllium neutron source", Health Phys. 7 (1962) 179-184.

(Su84) A. H. Sullivan, "The use of detectors based on ionization recombination in radiation protection”, CERN Report TIS-RP/130/CF, 30 April 1984. 
APPENDIX

ORIGINAL VERSION OF TM-1248

MARCH 1984 


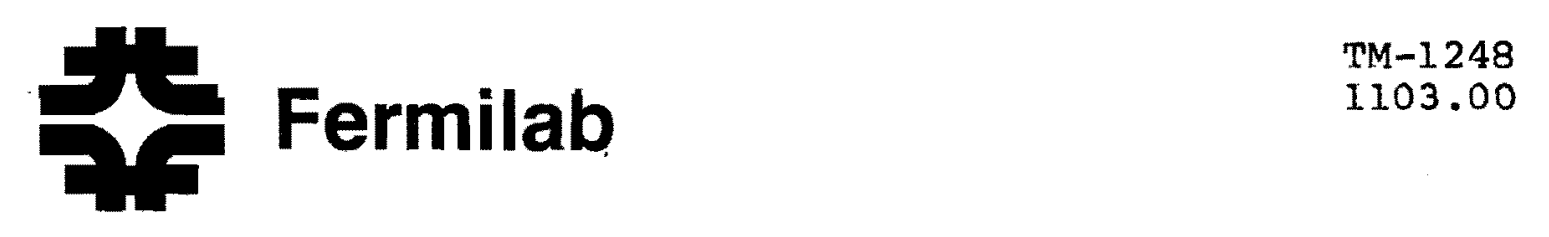

\author{
MEASUREMENTS OF RADIATION QUALITY FACTORS
}

USING A RECOMBINATION CHAMBER

J.D.Cossairt, D.W.Grobe, and M.A.Gerardi

March, 1984

\title{
1. INTRODUCTION
}

The measurement of dose equivalent rates in the presence of neutron radiation is complicated by the necessity to determine the quality factor(QF) of the radiation field. The $Q F$ is the absorbed dose of $200 \mathrm{keV}$ photons required to achieve the same biological effect as a unit absorbed dose of the subject field. It has been defined by the International Commission on Radiation Units and Measurements (IC7I) as an increasing function of linear energy transfer (LET). LET may, in essence, be operationally defined as the energy deposited by the radiation in a volume comparable to that of 
biological cells. Thus, energy which is carried out of this volume (e.g., delta rays) would be excluded but otherwise the concept of LET is closely related to the physical quantity $\mathrm{dE} / \mathrm{dx}$.

A variety of experimental techniques have been used to determine the quality factor of radiation fields ranging from direct spectrum measurements, which allow one to determine the average quality factor by performing integrations over the energy variable, to direct measurements of LET spectra in the so-called Rossi chamber(Ro68). Presented here is our experience with a recombination chamber. This technique has been described in a CERN report by Sullivan and Baarli (Su63) and summarized in the classic text of Patterson and Thomas (Pa73). In this method, one uses the fact that a high pressure ion chamber operated at a voltage beneath its plateau will measure a greater response to radiation of low LET than to radiation of high LET in fields of equivalent absorbed dose rate. Qualitatively, this results from the fact that the high LET radiation deposits the same energy per gram in dense tracks or clusters so that in the presence of a weak electric field, the migration time of the ions is long enough to allow for them to recombine. For low LET radiation, this columner recombination is much smaller because of the lower density of the ionization(the elections and ions are, on average, further apart) 
In the paper by Sullivan and Baarli, the following formula is quoted as describing the response of the chamber as a function of voltage:

$$
I=k V^{N}
$$

where $I$ is the normalized current collected by the chamber, $K$ is a constant of proportionality, $\mathrm{V}$ is the applied high voltage, and $\mathrm{N}$ is a fractional power which can be related to the LET and hence the quality factor of the radiation field. These workers also present a correlation between $\mathrm{N}$ and $\mathrm{QF}$ based upon measurements in radiation fields of known quality factor. The rest of this note describes a calibration procedure and results obtained in accelerator radiation fields.

\section{CALIBRATION PROCEDURE}

The Fermilab Safety Section possesses a chamber especially designed for doing recombination measurements ${ }^{1}$. Using this chamber the procedure is to measure its response over its range of operating potentials (up to 1200 volts) by collecting the liberated charge at the anode using a sensitive electrometer such as a Keithley $610 \mathrm{C}$. Equation (1) can be fit to the data using the least squares procedure to obtain a value of $\mathrm{N}$ in a field of known QF. For this particular chamber, this was done for several fields of known quality factor. A $\mathrm{QF}=1$ field from a radioactive source $\left({ }^{60} \mathrm{Co}\right)$ was used while for a 
$\mathrm{QF}=6.9 \mathrm{field}, \mathrm{a}{ }^{238} \mathrm{Pu}-\mathrm{Be}$ source was used. The value of 6.9 comes from the work of Hobffert and Raffnsфe(Ho80) and, of necessity, includes the contribution of gamma rays emitted from this source. It is thus smaller than the value for just the neutron component. Values of QF intermediate between these two were obtained by mixing the radiation field artificially using sources of various strengths. The quality factor of the mixed field was determined by averaging the absorbed dose rates due to individual sources. In all cases, the gamma and neutron sources were placed very close to each other so that the detector $(60 \mathrm{~cm}$ away) would be subjected to equivalent field nonuniformities from both sources. This measurement was done in the second floor of a brick house. The effect of scattering from the walls upon both the dose equivalent rate and the flux was calculated using the method of Jenkins (Je80) and found to be negligible for both quantities (less than 4 per cent). The response curves of chamber at different values of quality factor are displayed in Figure 1 along with the fits using $\mathrm{Eq}(1)$. These measurements were made in fields having absorbed dose rates ranging between 4.8 and 34 mrad per hour.

In this figure one sees that Eq(1) provides a very good $f i t$ to the response curves to first approximation. It does slightly overestimate the response at both the lowest potential(20 volts) and at the nominal saturation potential (1200 volts). Taking the values of $\mathrm{N}$ determined in this manner and plotting these as a function of $\mathrm{QF}$ in Figure 2, one sees a relationship quite similar to that reported by 
Sullivan and Baarli. At this point two different relationships were used to determine a functional relationship N(QF), linear and power law, and these are shown on Figure 2. Both of these are adequate fits to the data for $2<Q F<7$ which spans the range of quality factor values expected in the neutron fields produced by the Fermilab synchrotrons. For $Q F<2$, the errors are large since the technique requires one to measure a much smaller effect as a function of voltage (small $\mathrm{N}$ value). For small values of $\mathrm{N}$, it is better to make comparisons directly to the calibration measurements. Such a situation might be expected in a radiation field which is dominated by muons.

\section{MEASUREMENTS IN THE LABYRINTH AT NW3 (1984)}

The chamber was used to measure the quality factor in the labyrinth in enclosure NW3 shown in Figure 3. An aluminum target beneath the floor of this labyrinth $30 \mathrm{~cm}$ long and of square cross section (15 x $15 \mathrm{~cm}$ ) was struck by $400 \mathrm{GeV}$ protons in a beam spot having a FWHM of about $1.4 \mathrm{~cm}$ (both coordinates) at an intensity of 2 $x 10^{11}$ per spill. The two locations $A$ and $B$ were those at which the recombination chamber was used to make these measuremtents. Response curves similar to the ones shown in Figure 1 was obtained at these two locations, using a tissue equivalent ion chamber (Fermilab chupmunk, using the outputs of the charge digitizer) for normalization. The results are shown on Figure 4. An attempt was 
made to perform the normalization using the secondary emission monitor in this beamline directly, but the results were much less satisfactory due to short term beam intensity fluctuations not registered by the distant SEM (500 m upstream). The instantaneous absorbed dose rates (during a $15 \mathrm{sec}$. Energy Saver Spill) were about 0.13 and $0.016 \mathrm{mrad} / \mathrm{sec}$ at locations $A$ and $B$, respectively.

In Figure 4, we see that one can determine the quality factors to reasonable accuracy using this procedure. The result that the quality factor in the first leg of the labyrinth is larger than the value found in the second leg is not surprising since in the first leg, one would expect a more energetic neutron spectrum. The value found in the second $\mathrm{leg}$ is consistent with the result of a neutron spectrum measurement by J.Couch, A.Elwyn and W.Freeman (to be reported elsewhere).

4. MEASUREMENTS ABOUT ENCLOSURE EEl(1980)

Prior to the modifications to this enclosure in preparation for the Tevatron Wide Band Neutral Beam Project, EEl had a very thinly shielded roof which was the source of considerable radiation during $400 \mathrm{GeV}$ operations. Figure 5 shows this enclosure along with two locations of recombination chamber measurements ( $C$ and $D$ ). The instantaneous absorbed dose rates (during a 1 sec. Main Ring spill) were about 0.8 and $0.04 \mathrm{mrad} / \mathrm{sec}$ at locations $C$ and $D$, respectively. 
At the upstream end of the roof of EEl the absorbed dose rate was predominantly due to neutrons while at the downstream end, the absorbed dose rate was predominantly muons(Co83). The response curves measured for the chamber in these two locations are shown in Figure 6 along with the fits to the data using the procedure described above. The data values were normalized to the P-East SEM at these two locations. As one can see in these results, the quality factor of 4.5 measured at location $C$ is consistent with the general "rule of thumb" value of five predicted by Patterson(Pa71). This agrees with a value of four found in approximately the same location by R.V.Griffith(Gr81) who used a multisphere technique to measure the neutron energy spectrum. At the downstream end (location D), the result is consistent with unity, expected in this field which is known to be predominantly muons. These two measurements could likely have been improved by normalizing to the digital output of a nearby detector, rather than to a somewhat distant SEM.

\section{CONCLUSION}

It is concluded that the recombination chamber isauseful instrument for determine the approximate quality factor in mixed fields of radiation and may be confidently used in conjunction with other appropriate techniques. The method of measuring the response curve of the chamber appears to be a reasonable approach if one fits the data using $\mathrm{Eq}(1)$. One must be careful to choose a method of 
normalizing the data which is adequate. It appears that the best procedure is to normalize to a nearby detector having a digital readout.

We acknowledge the help of s.Velen, A.Elwyn, and W.Freeman during these measurements.

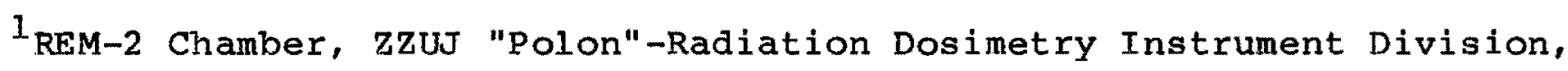
Bydgoszcz, Poland. 
REFERENCES

Co83 J.D.Cossairt and L.V.Coulson, "Neutron Skyshine Measurements at Fermilab" FN-394(1983), to be published in Health Phys.

Gr81 R.V.Griffith, Lawrence Livermore National Laboratory, private communication (1981).

Ho80 M.Hôfert and C.Raffns $\varnothing$, "Measurement of Absolute Absorbed Dose and Dose-Equivalent Response for Instruments Used Around High Energy Proton Accelerators, Nucl. Instr. and Meth. $176(1980) 443$.

IC71 Radiation Quantities and Units, ICRU Report 19(ICRU, Washington, 1971).

Pa71 H.W.Patterson, J.T.Routti, and R.Thomas, "What Quality Factor?", Health Phys.20 (1971) 517.

Pa73 H.Wade Patterson and Ralph H.Thomas, Accelerator Health Physics (Academic Press, NY 1983).

Ro68 H.H.Rossi, "Microscopic Energy Distributions in Irradiated Matter", P.43 Radiation Dosimetry, Vol I, Second Edition, F.H.Attix, Ed. (academic Press,NY, 1968).

Su63 A.H.Sullivan and J.Baarli, "An Ionization Chamber for the Estimation of the Biological Effectiveness of Radiation", CERN Report No.63-17(European Organization for Nuclear Research, Geneva, 1963). 
100

1000

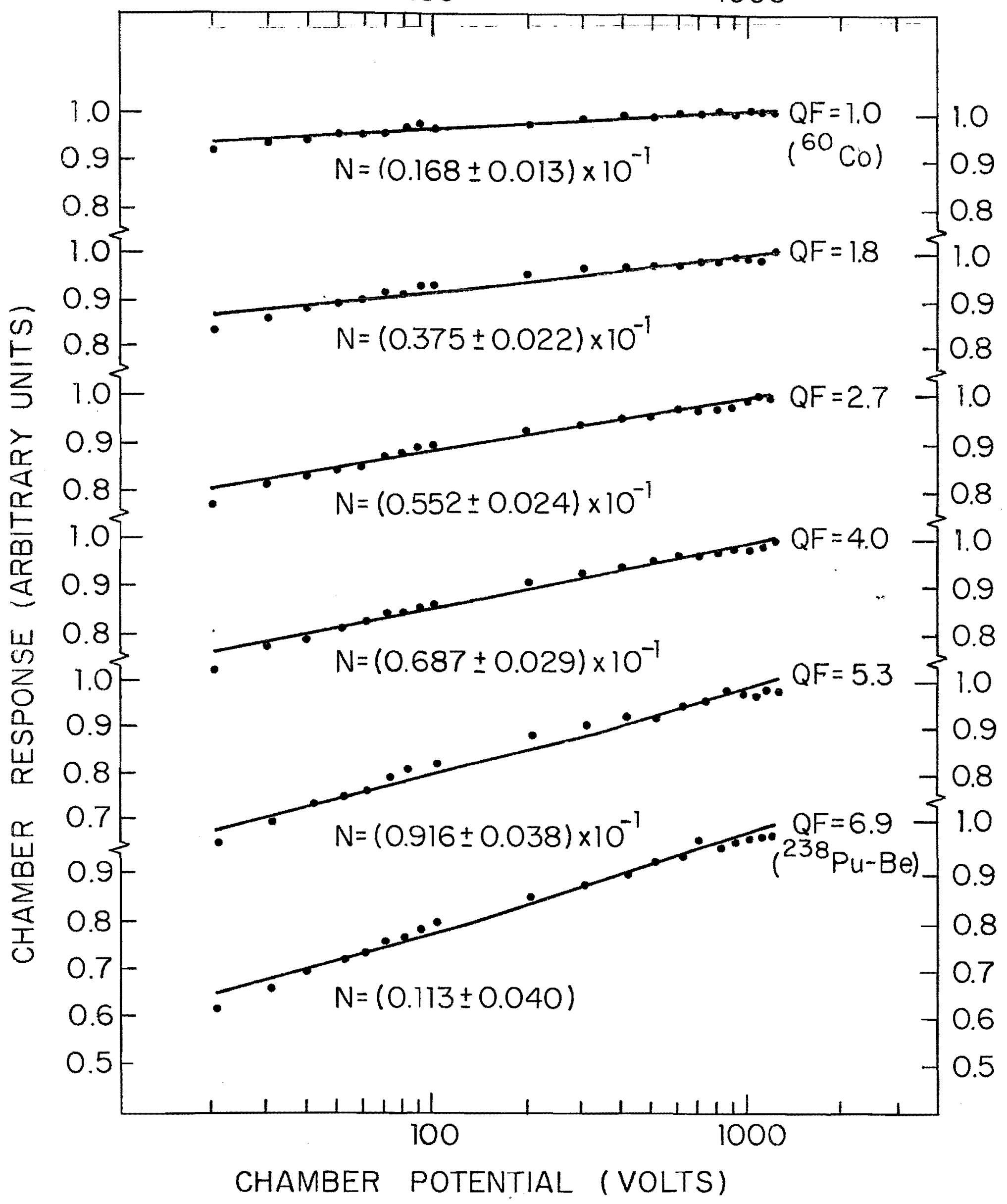

FIGURE I 


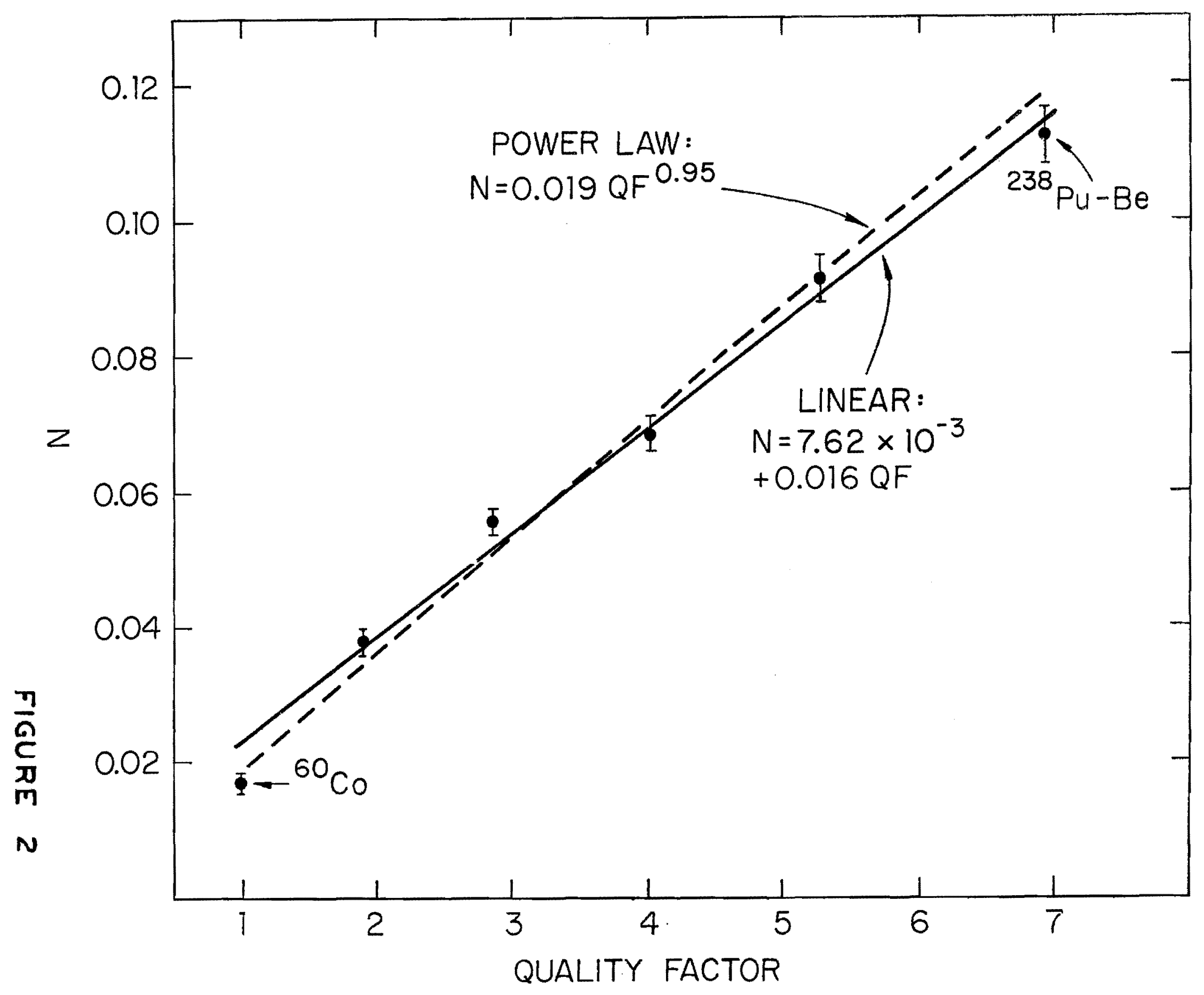




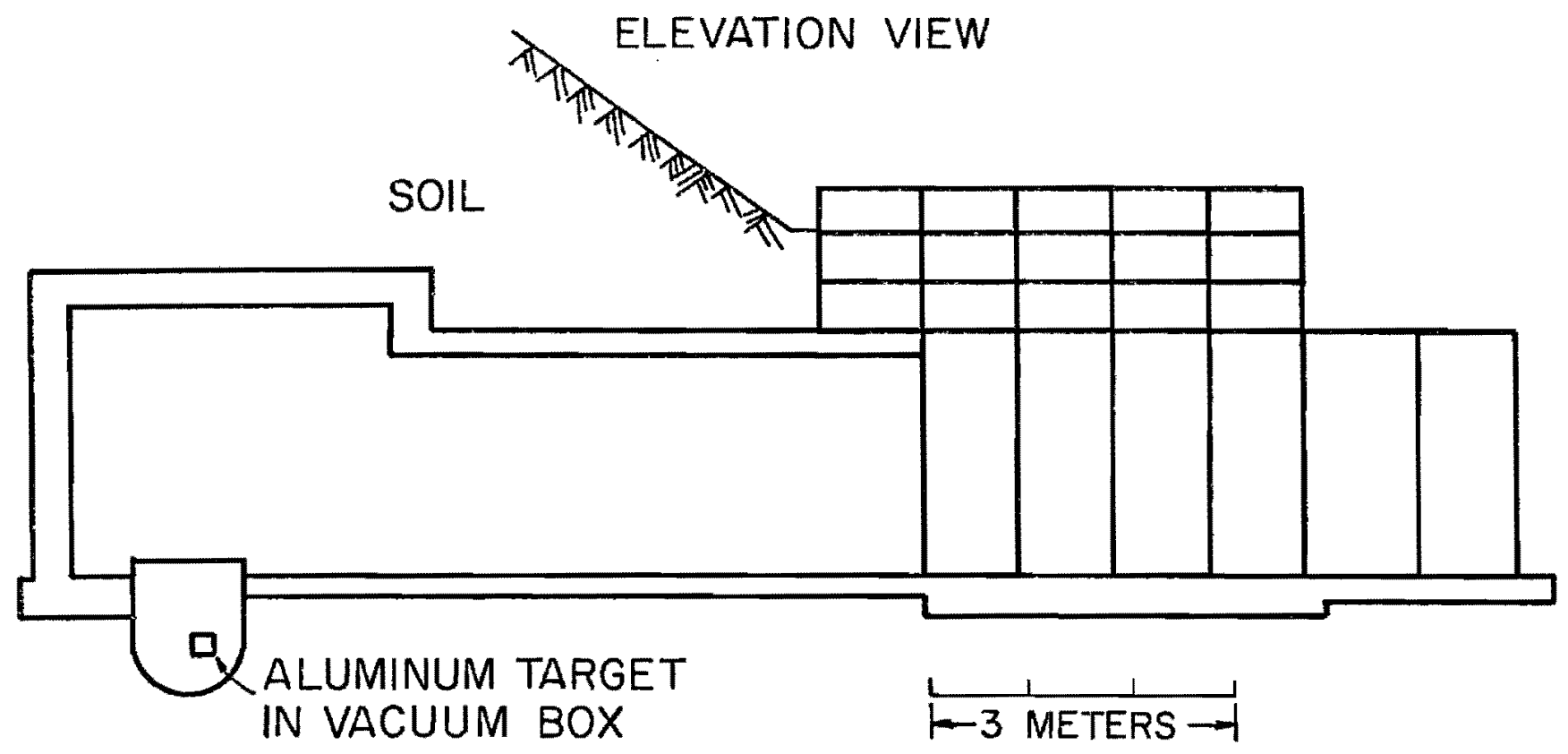

\section{PLAN VIEW}

400 GEV PROTONS
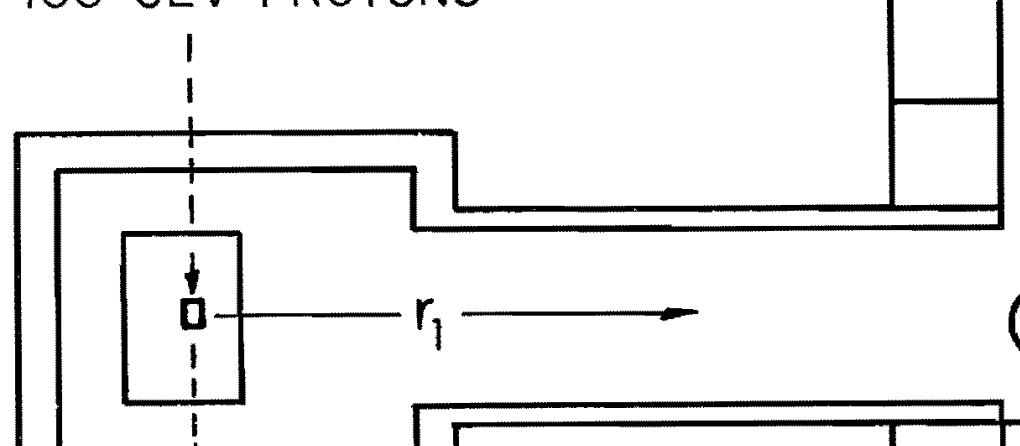

(A)

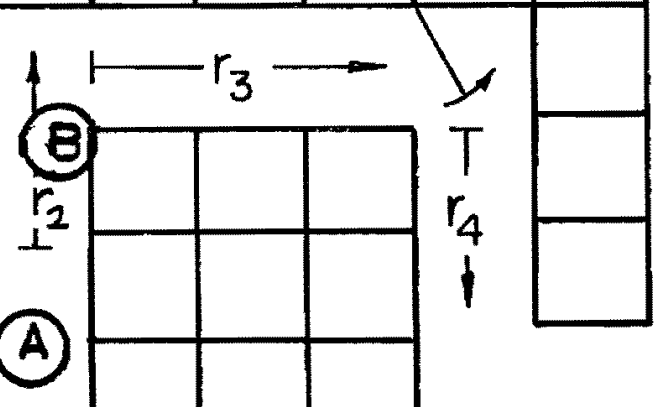

CONCRETE SHIELDING BLOCKS 
100

1000

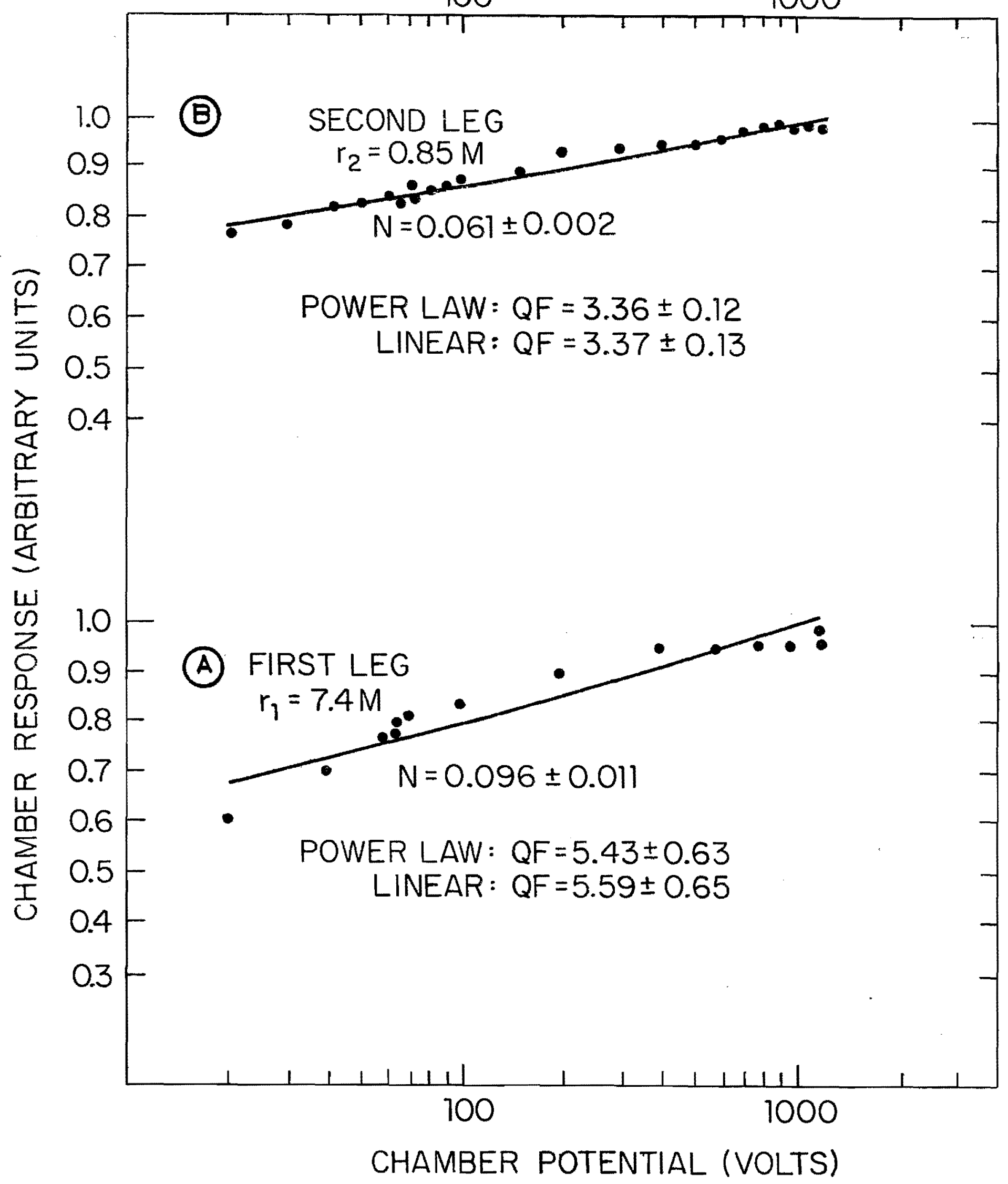

FIGURE 4 


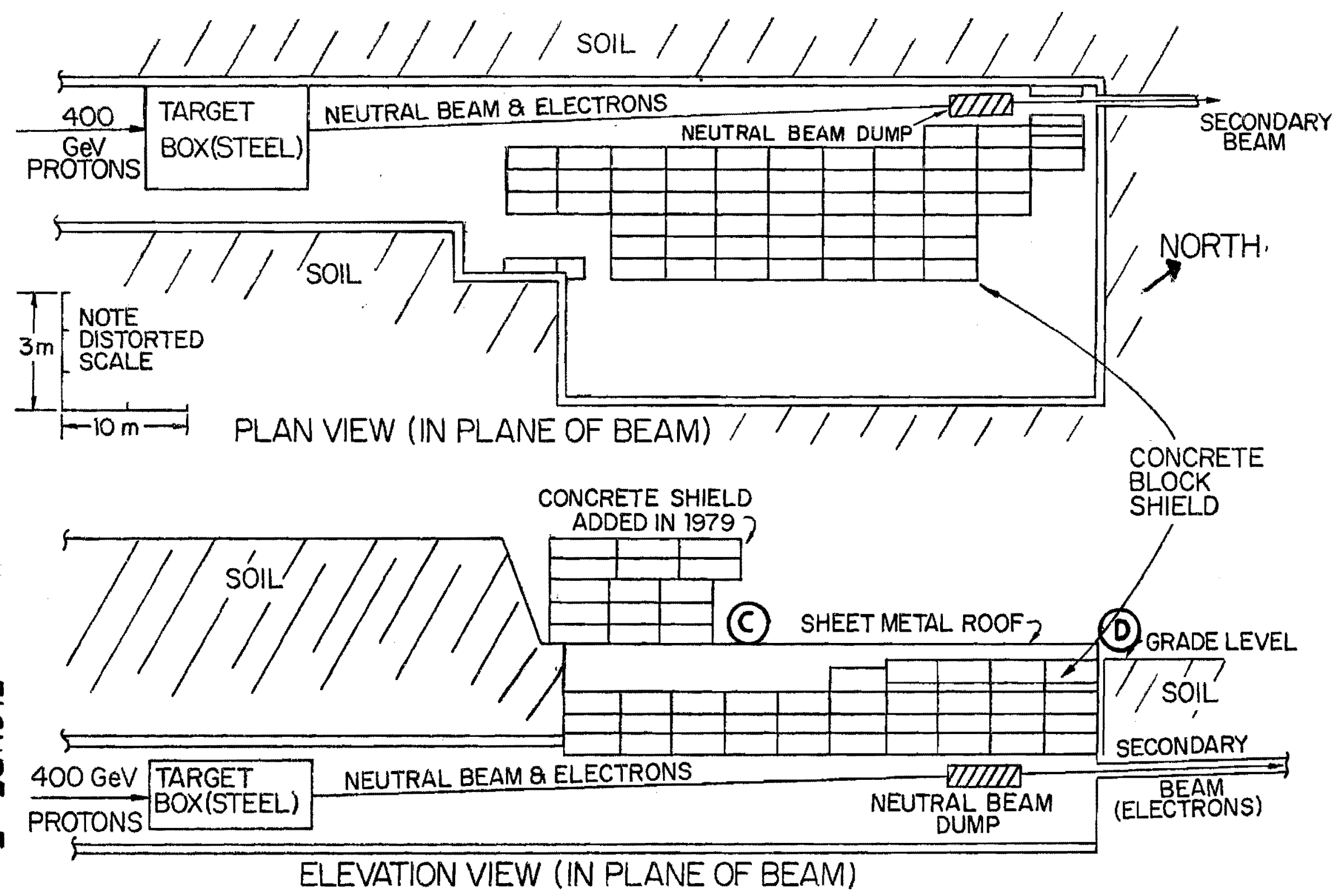




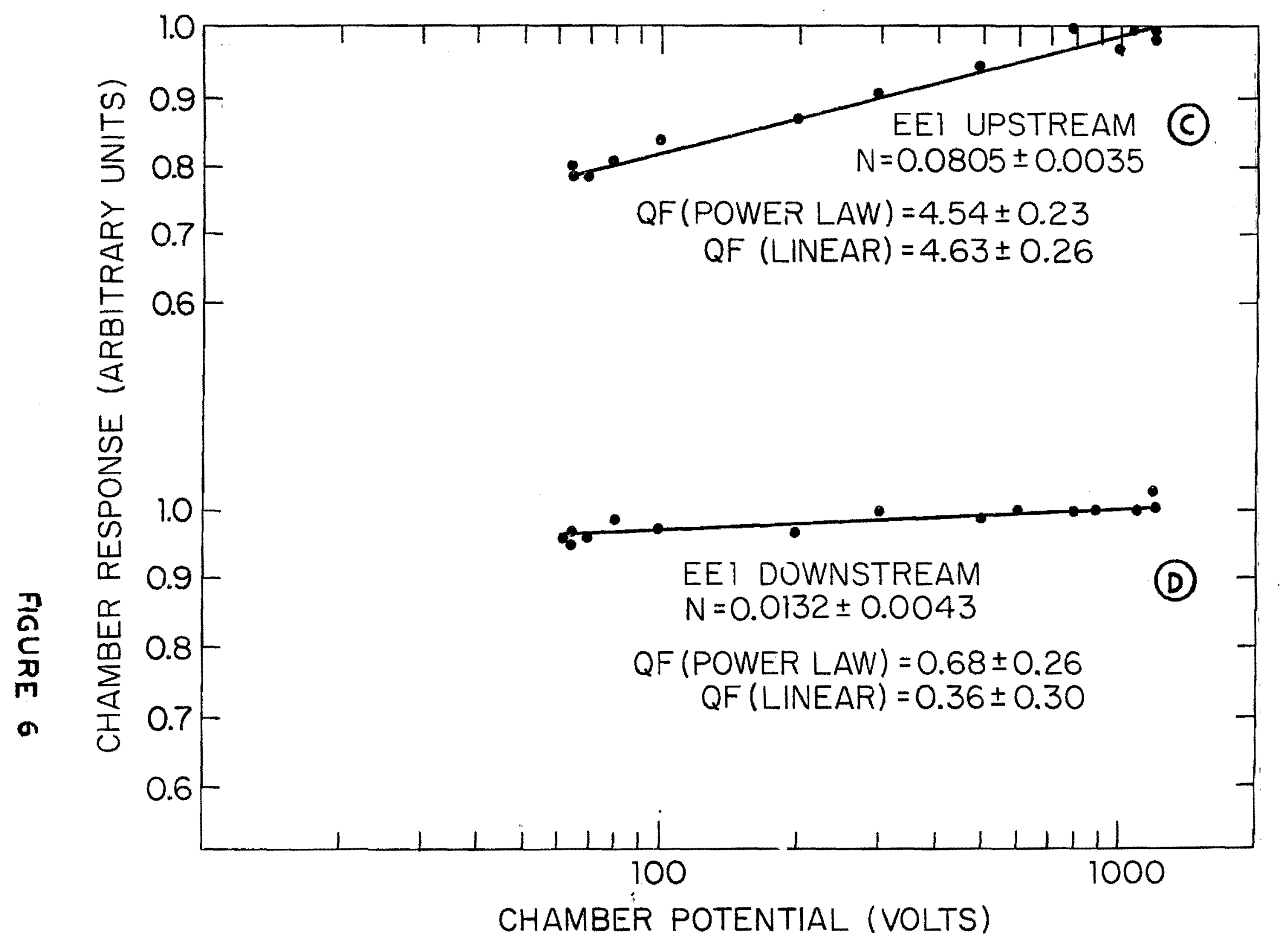

